\title{
Mutant prevention concentration of colistin alone and in combination with rifampicin for multidrug-resistant Acinetobacter baumannii
}

\author{
H. Nordqvist ${ }^{1,2}$ (I) $\cdot$ L. E. Nilsson ${ }^{3} \cdot$ C. Claesson ${ }^{3}$
}

Received: 9 May 2016 / Accepted: 17 July 2016/Published online: 10 August 2016

(C) The Author(s) 2016. This article is published with open access at Springerlink.com

\begin{abstract}
Colistin-susceptible isolates of Acinetobacter baumannii often contain subpopulations that are resistant to colistin. Monotherapy with colistin can lead to selective growth of these subpopulations and emergence of colistin-resistant strains. Our objectives were to explore the susceptibility pattern of colistin-resistant subpopulations and investigate if combining colistin with a second antibiotic could prevent their selective growth. Four colistin-susceptible clinical isolates of A. baumannii and one reference isolate were used. The mutant prevention concentration (MPC) of colistin, i.e. the concentration required to block growth of all single-step-mutant subpopulations, was determined by plating an inoculum of $10^{9} \mathrm{CFU}$ on Mueller Hinton agar (MHA)-plates containing 2-fold dilutions of colistin $(0.125-128 \mathrm{mg} / \mathrm{L})$. Susceptibility testing of colistinresistant subpopulations, obtained in the MPC assay, was performed with Etest. The MPC of colistin, in combination with rifampicin, was determined by plating an inoculum of $10^{9} \mathrm{CFU}$ on MHA-plates containing colistin $(0.125-128 \mathrm{mg} / \mathrm{L})$ and fixed concentrations of rifampicin $(1.1 \mathrm{mg} / \mathrm{L}$ or $4.4 \mathrm{mg} / \mathrm{L})$. The colistin-resistant subpopulations demonstrated increased susceptibility to a number of agents compared to their main populations. These subpopulations were even susceptible to agents
\end{abstract}

H. Nordqvist

hampus.nordqvist@sodersjukhuset.se

C. Claesson

carina.claesson@liu.se

1 Department of Infectious Diseases, Linköping University Hospital, Linköping, Sweden

2 Present address: Department of Infectious Diseases, Stockholm South Hospital, Stockholm, Sweden

3 Clinical Microbiology, Department of Clinical and Experimental Medicine, Linköping University, Linköping, Sweden that normally are inactive against gram-negative bacteria and all had rifampicin MICs of $<0.002 \mathrm{mg} / \mathrm{L}$. The combination of colistin and rifampicin completely inhibited the growth of all colistin-resistant subpopulations and significantly lowered the MPC of colistin for A. baumannii. Combining colistin with rifampicin could be a way to prevent selective growth of colistin-resistant subpopulations of A. baumannii and possibly the emergence of colistin-resistant strains.

\section{Introduction}

Acinetobacter baumannii has emerged as an important cause of nosocomial infections. Its clinical significance is primarily related to its remarkable ability to upregulate and acquire resistant determinants, making it notoriously difficult to treat [1]. Surveillance studies now show that $>80 \%$ of invasive isolates of A. baumannii are carbapenem resistant in some European countries [2].

Colistin is an antibiotic with bactericidal effect against gram-negative bacteria. It is often used as a last resort treatment for multi drug-resistant (MDR) gram-negative bacteria, including carbapenem-resistant A. baumannii [3]. Unfortunately, colistin-resistant strains of $A$. baumannii have emerged $[4,5]$. As a consequence we now face the risk of infections caused by A. baumannii that cannot be treated with antimicrobials.

Heteroresistance to colistin due to resistant subpopulations is a well-described phenomenon among A. baumannii [4]. Colistin-resistant strains can develop if the colistin concentration is sufficient to prevent growth of the susceptible main population but not the resistant subpopulations [6]. The concentration required to prevent such selective growth is called the mutant prevention concentration (MPC). The MPC is 
defined as the concentration required to block growth of all single-step-mutant subpopulations $[7,8]$.

The MPC of colistin in vitro for A. baumannii has been shown to be so high that it cannot be exceeded in vivo. These results suggest that monotherapy with colistin will inevitably lead to selective growth of colistin-resistant subpopulations. The MPC of colistin against A. baumannii was lowered when colistin was combined with levofloxacin or tobramycin. It was however still well beyond clinically achievable concentrations $[9,10]$. The results from one study implicate that other agents, for example, rifampicin and meropenem, may be more effective in lowering the MPC [11].

Our first objective was to explore the susceptibility pattern of colistin-resistant subpopulations of A. baumannii. Our second objective was to investigate if we could prevent their selective growth by combining colistin with a second antibiotic that the colistin-resistant subpopulations were susceptible to.

\section{Material and methods}

Characterization of bacterial isolates Four clinical isolates (AB1-AB4) of A. baumannii isolated from four different patients during 2013 at the Department of Clinical Microbiology, Linköping University Hospital (Linköping, Sweden) were included. Species identification was done with MaldiTof (Bruker Daltonics Scandinavia AB, Solna Sweden). Pulse-field gel electrophoresis (PFGE) and determination of the resistance genes present in AB1-AB4 were performed at the Public Health Agency of Sweden (Solna, Sweden). According to PFGE the four isolates represented four different clones. The following carbapenemase genes were identified: OXA 23 (AB1-AB3), OXA 51 (AB1-AB4), OXA 58 (AB4) and NDM (AB1). All clinical isolates were defined as MDR according to proposed nomenclature [12], but classified as susceptible to colistin ( $\mathrm{S} \leq$ $2 \mathrm{mg} / \mathrm{L}$ ) [13]. A. baumannii CCUG 19096 (AB5), Pseudomonas aeruginosa ATCC 27853 and Escherichia coli ATCC 25922, obtained from the Culture Collection, Göteborg University, Sweden, were used as type strains.

MIC of colistin determined with Etest, broth dilution and agar dilution Colistin MIC was determined for isolates AB15 with Etest (bioMérieux, Marcy l'Etoile, France), broth dilution and agar dilution. Colistin Etest was applied on MuellerHinton Agar (MHA) (Becton Dickinson, Franklin Lakes, NJ, USA) inoculated with a bacterial suspension of $0.5 \mathrm{McFarland}$ in $0.85 \% \mathrm{NaCl}$ and was read after $24 \mathrm{~h}$ incubation at $36{ }^{\circ} \mathrm{C}$. Broth dilution MIC of colistin sulphate (Sigma-Aldrich corp., St Louis, MO, USA) was determined with 2-fold dilutions $(0.016-256 \mathrm{mg} / \mathrm{L})$ in glass tubes containing $1 \mathrm{~mL}$ MuellerHinton Broth (MHB) (Becton Dickinson), with an inoculum of approximately $1 \times 10^{5} \mathrm{CFU} / \mathrm{mL}$. Broth dilution MIC was defined as the lowest concentration inhibiting visible growth after $24 \mathrm{~h}$ of incubation at $36{ }^{\circ} \mathrm{C}$. Agar dilution MIC was determined with MHA (Becton Dickinson) plates with 2fold dilutions of colistin sulphate (Sigma Aldrich), ranging from 0.08 to $2.0 \mathrm{mg} / \mathrm{L}$. A bacterial suspension of approximately $10^{5} \mathrm{CFU} / \mathrm{mL}$, determined by viable count, was prepared by dilution with $\mathrm{NaCl} 0.85 \%$ of an overnight culture of $3 \mathrm{~mL}$ MHB (Becton Dickinson). Plates were spot inoculated, with two spots and $10^{4} \mathrm{CFU}$ in each spot. The plates were incubated overnight at $36^{\circ} \mathrm{C}$. Agar-MIC was defined as the lowest concentration that inhibited visible growth. All tests were performed in duplicates to ensure reproducibility.

MPC of colistin determined with agar dilution The MPC was determined with a method described by Cai et al. [9], but with modifications. Isolates AB1-5 were grown overnight on Columbia agar (Acumedia, Lansing, MI, USA) with $5 \%$ defibrinated horse blood (Håtuna, Uppsala, Sweden) at $36{ }^{\circ} \mathrm{C}$. Glass tubes containing $2 \mathrm{~mL}$ MHB (Becton Dickinson) were inoculated with bacteria from the Columbia agar and incubated overnight at $36^{\circ} \mathrm{C}$ with shaking, reaching a bacterial concentration of $10^{9} \mathrm{CFU} / \mathrm{mL}$, determined by viable count. Aliquots of $1 \mathrm{~mL}$ were plated on MHA-plates (Becton Dickinson) containing colistin sulphate (Sigma Aldrich) in 2-fold dilutions $(0.125-128 \mathrm{mg} / \mathrm{L})$. The MPC was defined as the lowest concentration of colistin that inhibited all visible growth of $10^{9} \mathrm{CFU}$ after $48 \mathrm{~h}$ of incubation [7]. Colonies were isolated from the plates with the highest and the second highest colistin concentration with growth of bacteria. MaldiTof (Bruker) was used to determine that the colonies were A. baumannii, and Etest (bioMérieux) was used to determine their susceptibility to colistin. The tests were performed in duplicates to ensure reproducibility.

MIC of the main populations (AB1-5) and the colistinresistant subpopulations determined with Etest The main populations of the isolates AB1-5 and the colistin-resistant subpopulations isolated from the colistin plates in the MPC assay were studied further. The MIC of colistin, vancomycin, rifampicin, meropenem, linezolid, ampicillin/sulbactam, tigecycline, ciprofloxacin and tobramycin was determined with Etest (bioMérieux) on MHA-plates (Becton Dickinson) inoculated with a bacterial suspension adjusted to $0.5 \mathrm{McF}$ arland in $0.85 \% \mathrm{NaCl}$. The MIC was read after $24 \mathrm{~h}$ of incubation at $36{ }^{\circ} \mathrm{C}$ for vancomycin and colistin and after $18 \mathrm{~h}$ for the remaining agents.

MPC of colistin in combination with rifampicin determined with agar dilution Bacterial suspensions with a concentration of $10^{9} \mathrm{CFU} / \mathrm{mL}$ were generated for isolate $\mathrm{AB} 1$ $\mathrm{AB} 5$ as described above in the method for the MPC of colistin determined with agar dilution. Aliquots of $1 \mathrm{ml}$ were plated on MHA-plates (Becton Dickinson) containing colistin sulphate 
Table 1 MIC determination of colistin with Etest, agar and broth dilution assay

\begin{tabular}{llll}
\hline Isolate & Etest MIC $(\mathrm{mg} / \mathrm{L})$ & Agar dilution MIC $(\mathrm{mg} / \mathrm{L})$ & Broth dilution MIC $(\mathrm{mg} / \mathrm{L})$ \\
\hline $\mathrm{AB} 1$ & 0.125 & 0.5 & 1 \\
$\mathrm{AB} 2$ & 0.125 & 0.5 & 1 \\
$\mathrm{AB} 3$ & 0.125 & 0.25 & 1 \\
$\mathrm{AB} 4$ & 0.125 & 0.25 & 0.5 \\
$\mathrm{AB} 5$ & 0.125 & 0.5 & 1 \\
\hline
\end{tabular}

(Sigma Aldrich) $0.125-128 \mathrm{mg} / \mathrm{L}$ (using 2-fold dilutions) in combination with fixed concentrations of rifampicin (Sigma Aldrich) 1.1 or $4.4 \mathrm{mg} / \mathrm{L}$. We choose concentrations of rifampicin that we considered clinically relevant. Free unbound concentration of $1.1 \mathrm{mg} / \mathrm{L}$ can readily be achieved with per oral administration of $600 \mathrm{mg}$ rifampicin and $4.4 \mathrm{mg} / \mathrm{L}$ with $900 \mathrm{mg}[14,15]$. The tests were performed in duplicates to ensure reproducibility. The MPC was defined as the lowest concentration of colistin that inhibited all visible growth of an inoculum of $10^{9} \mathrm{CFU}$ after $48 \mathrm{~h}$ of incubation at $36^{\circ} \mathrm{C}$ [7]. Colonies were isolated from the plates with the highest and the second highest colistin concentration with growth of bacteria. MaldiTof (Bruker) was used to determine that the colonies were A. baumannii and Etest (bioMérieux) to determine their susceptibility to colistin and rifampicin.

\section{Results}

Colistin MIC The MIC of colistin for the main population determined with Etest, agar dilution and broth dilution were $0.125,0.25-0.5$ and $0.50-1 \mathrm{mg} / \mathrm{L}$, respectively (Table 1 ).

MPC of colistin alone The MPC was $64 \mathrm{mg} / \mathrm{L}$ for all isolates except AB3 that displayed an MPC-value of $16-32 \mathrm{mg} / \mathrm{L}$ (Table 2). Colistin-resistant subpopulations were recovered from MHA-plates containing 16-32 $\mathrm{mg} / \mathrm{L}$ colistin for all tested isolates in the frequency of 1 of $10^{7}-10^{9}$, except for AB3 from which no colistin-resistant subpopulations were recovered. Subpopulations displaying colistin-dependent growth were also recovered from colistin-containing plates for isolates $\mathrm{AB} 1$ and $\mathrm{AB} 2$ (Fig. 1a). Colistin-susceptible cells were detected on plates containing colistin $8-32 \mathrm{mg} / \mathrm{L}$ in all isolates. They were most often found co-existing with colistinresistant subpopulations, but not always.

Antibiograms for main populations and colistin-resistant subpopulations MIC values with Etest were determined for the main populations and the colistin-resistant subpopulations (Table 3). The main population of four isolates (AB1-4) were MDR [12] with high MIC values for all antimicrobial agents tested, except for colistin. The main population of one isolate displayed high-level resistance to rifampicin $>32 \mathrm{mg} / \mathrm{L}$ (AB3) while the other isolates' main populations had MIC values of 4-8 mg/L. The colistin-resistant subpopulations clearly displayed increased susceptibility to rifampicin, vancomycin and meropenem compared to the main populations. The rifampicin MICs were much lower $(<0.002 \mathrm{mg} / \mathrm{L})$ for all colistin-resistant subpopulations compared to the main populations (Fig. 1b).

MPC of colistin in combination with rifampicin We chose to combine colistin with rifampicin because of the results in the antibiogram study described above. The MPC-values of colistin when combined with rifampicin at fixed concentrations of $1.1 \mathrm{mg} / \mathrm{L}$ or $4.4 \mathrm{mg} / \mathrm{L}$ are reported in Table 2 . The MPC was lowered from $64 \mathrm{mg} / \mathrm{L}$ to $8-16 \mathrm{mg} / \mathrm{L}$ for the isolates $\mathrm{AB} 1, \mathrm{AB} 2, \mathrm{AB} 4$ and AB5 when colistin was combined with rifampicin $1.1 \mathrm{mg} / \mathrm{L}$ or $4.4 \mathrm{mg} / \mathrm{L}$. The MPC was not lowered for the AB3 isolate. No difference regarding the MPC could be seen when comparing plates containing rifampicin $1.1 \mathrm{mg} /$ $\mathrm{L}$ and rifampicin $4.4 \mathrm{mg} / \mathrm{L}$. Interestingly, no colistin-resistant subpopulations were detected on any of the MHA plates containing both colistin and rifampicin $1.1 \mathrm{mg} / \mathrm{L}$ or $4.4 \mathrm{mg} / \mathrm{L}$. We
Table 2 Mutant prevention concentration (MPC) of colistin alone and in combination with rifampicin $1.1 \mathrm{mg} / \mathrm{L}$ or $4.4 \mathrm{mg} / \mathrm{L}$

\begin{tabular}{llll}
\hline Isolate & \multicolumn{2}{l}{ MPC $(\mathrm{mg} / \mathrm{L})$} & \\
\cline { 2 - 4 } & Colistin alone & Colistin $+1.1 \mathrm{mg} / \mathrm{L}$ rifampicin & Colistin $+4.4 \mathrm{mg} / \mathrm{L}$ rifampicin \\
\hline AB1 & 64 & $8-16$ & $8-16$ \\
AB2 & 64 & 16 & $8-16$ \\
AB3 & $16-32$ & $16-32$ & 32 \\
AB4 & 64 & 8 & 8 \\
AB5 & 64 & $8-16$ & $8-16$ \\
\hline
\end{tabular}



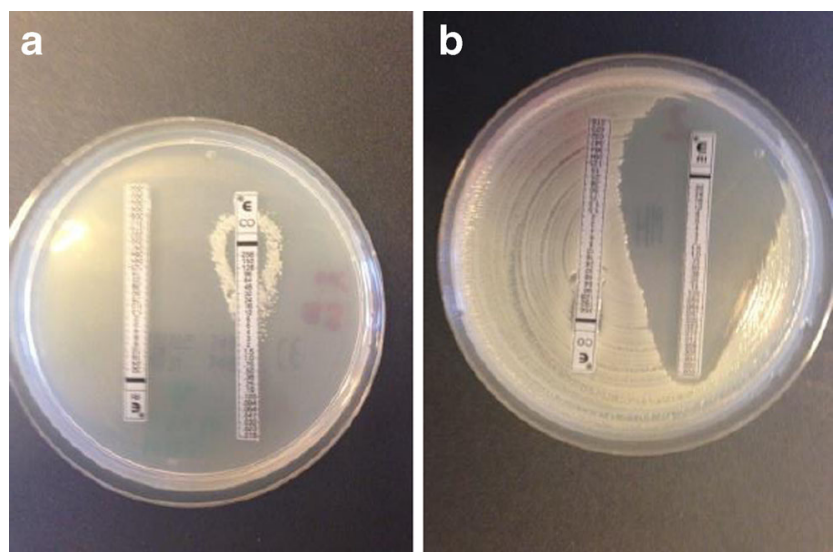

Fig. 1 MIC for rifampicin and colistin after overnight incubation on MHA. a Colistin-dependent growth - colonies growing only along the colistin Etest strip. b Colistin-resistant subpopulation with MIC for rifampicin $<0.002 \mathrm{mg} / \mathrm{L}$

detected colistin-susceptible cells with high-level resistance against rifampicin on MHA-plates containing both colistin and rifampicin in all isolates. No difference could be seen when comparing plates containing rifampicin $1.1 \mathrm{mg} / \mathrm{L}$ and $4.4 \mathrm{mg} / \mathrm{L}$.

\section{Discussion}

Colistin-resistant strains of A. baumannii can develop during colistin therapy if colistin-resistant subpopulations grow selectively [16-19]. We hypothesized that such growth could be prevented if colistin was combined with an agent effective against these subpopulations. The colistin-resistant subpopulations were extremely susceptible to rifampicin (MIC < $0.002 \mathrm{mg} / \mathrm{L}$ ) and the combination of colistin and rifampicin completely inhibited the growth of all colistin-resistant subpopulations and significantly lowered the MPC of colistin.

The colistin-resistant subpopulations demonstrated increased susceptibility to a number of agents compared to their main population. They were even susceptible to rifampicin and vancomycin, agents that normally are inactive against gram-negative bacteria. These findings are likely due to increased outer membrane permeability, allowing greater access to target sites for these agents. One study reported similar results [11]. Meropenem and especially rifampicin demonstrated the greatest potential to prevent selective growth, with MICs for the colistin-resistant subpopulations of 0.016 $0.5 \mathrm{mg} / \mathrm{L}$ and $<0.002 \mathrm{mg} / \mathrm{L}$, respectively. Rifampicin has the advantage of not being nephrotoxic, but has the disadvantages of possible hepatotoxicity and drug interactions [20]. The broad spectrum of meropenem could be of benefit since many patients infected with A. baumannii are co-infected [20], while disadvantages are possible nephrotoxicity when it is combined with colistin [21] and the risk of an increase in carbapenem resistance if carbapenem consumption is increased [22].

We chose to combine colistin with rifampicin because of the colistin-resistant subpopulations extreme susceptibility to this agent. To our knowledge no previous study has investigated the effect on the MPC of colistin for A. baumannii when rifampicin is added. In our study the MPC of colistin was lowered $\geq 2$ dilution steps in all heteroresistant isolates when rifampicin was added in clinically achievable concentrations. No colistin-resistant subpopulations were recovered from any MHA-plates containing both colistin and rifampicin. These results show that rifampicin can prevent selective growth of colistin-resistant subpopulations in vitro. Our results are supported by other in vitro studies $[19,23]$. Most in vitro studies have shown $100 \%$ synergy for the colistin-rifampicin combination against MDR A. baumannii with low to intermediate rifampicin resistance (MIC $\leq 16 \mathrm{mg} / \mathrm{L}$ ) [24, 25]. It is possible that a synergistic effect contributed to the lowered MPC seen in our heteroresistant isolates.

Evidence that rifampicin prevents the development of colistin-resistance in vivo is still lacking, although a colistinresistant strain of $A$. baumannii has successfully been treated with rifampicin [19]. We have found only one in vivo study that has investigated this issue (secondary outcome). No difference regarding colistin-resistance development could be seen when comparing colistin vs. colistin and rifampicin, since no development of colistin-resistance could be detected in any patient [20]. While our study and many in vitro studies $[9,10,16-18]$ indicate a very high risk of development of

Table 3 Etest MIC of main population and colistin-resistant subpopulations of isolates AB1-AB5

Isolate $\quad \mathrm{MIC}(\mathrm{mg} / \mathrm{L})$ of main population/colistin-resistant subpopulation

\begin{tabular}{lllllllll} 
& Colistin & Rifampicin & Vancomycin & Linezolid & Meropenem & Ciprofloxacin & Tigecycline & Tobramycin \\
\hline AB1 & $0.125 / 128$ & $8 /<0.002$ & $>256 / 0.125$ & $>256 / 32$ & $>32 / 0.064-0.25$ & $>32 / 15->32$ & $4 / 0.5$ & $12 / 0.5-2$ \\
AB2 & $0.125 / 12-128$ & $8 /<0.002$ & $>256 / 0.25-1$ & $>256 / 32-128$ & $>32 / 0.125-0.5$ & $>32 / \geq 32$ & $4 / 0.5-1$ & $2 / 0.5-2$ \\
AB3 & $0.125 /-$ & $>32 /-$ & $>256 /-$ & $>256 /-$ & $>32 /-$ & $>32 /-$ & $2 /-$ & $>256 /-$ \\
AB4 & $0.125 />256$ & $8 /<0.002$ & $>256 / 2$ & $>256 / 32$ & $4 / 0.064$ & $>32 />32$ & $4 / 1$ \\
AB5 & $0.125 / 16-32$ & $4 /<0.002$ & $>256 / 0.125$ & $>256 / 32$ & $1 / 0.016$ & $>32 / 0.032$ & $2 / 0.064$ & $4 / 0.064$ \\
\hline
\end{tabular}

For AB3 only Etest MIC of main population is presented, since no colistin-resistant subpopulations were detected in this isolate 
colistin-resistance with colistin alone, in vivo studies show conflicting data $[19,20,26]$. This discrepancy in vivo could be due to different length of follow up [20,26], the wide use of concomitant antibiotics in vivo [20], colistin dosage [20], reduced ability for the colistin-resistant organisms to survive and replicate in vivo [27] or overestimation of the MPC in vitro [7]. We believe that the MPC of colistin for A. baumannii is overestimated in vitro due to the inoculum effect for colistin [28]. No colistin-susceptible cells could be detected on plates containing $\geq 0.5 \mathrm{mg} / \mathrm{L}$ colistin when using an inoculum of $10^{5} \mathrm{CFU}$, but up to $32 \mathrm{mg} / \mathrm{L}$ with $10^{9} \mathrm{CFU}$.

No colistin-resistant subpopulations were detected in AB3 and the MPC of colistin alone was also 1-2 dilutions steps lower for this isolate. The MPC of colistin was not affected for AB3 when rifampicin was added. This was expected due to lack of colistin-resistant subpopulations and the high-grade rifampicin resistance (MIC $>16 \mathrm{mg} / \mathrm{L}$ ) displayed by $\mathrm{AB} 3$. High-grade rifampicin resistance is mediated by rpoB-mutations. No additive or synergistic effect is expected for the colistin-rifampicin combination against isolates carrying this mutation [25]. To our knowledge no correlation has been found between rpoB-mutation and lack of colistin-resistant subpopulations. We therefore believe that the combination of findings in $\mathrm{AB} 3$ were a mere coincidence.

We did not detect any colistin-resistant cells with rifampicin resistance, but development of resistance due to rpoB mutations is always a concern when using rifampicin. This mutation can occur spontaneously or upon rifampicin exposure and colistinsusceptible A. baumannii, like AB3, readily acquire it [20]. Compared to the main populations, colistin-resistant subpopulations exist in substantially lower numbers $[8,19]$ and are significantly more susceptible to rifampicin [11]. It is therefore less likely that the colistin-resistant subpopulation would mutate in the rpoB gene spontaneously or upon rifampicin exposure compared to the colistin-susceptible main population.

Colistin susceptibility testing is problematic and disconcordant results for different susceptibility testing methods are a well-known problem [29-32]. The categorical agreement was $100 \%$ for Etest, agar and broth dilution in our study. Only Etest was used for colonies isolated in the MPC-study to differentiate between colistin susceptible and resistant cells. This method was chosen for practical reasons and because very good performance has been demonstrated with Etest for colistin [29, 30]. However, a risk of Etest falsely classifying isolates as colistin susceptible has been demonstrated. We had no Etest results in the range $(0.5-2.0 \mathrm{mg} / \mathrm{L})$ for which the absolute majority of these erroneous results have been demonstrated [31, 32]. We therefore assess that the likelihood for false susceptible results in our study is low.

One interesting finding in our study was subpopulations that exhibited a colistin-dependent growth pattern. This phenomenon has previously been described for colistin and A. baumannii. The authors suggest that one possible mechanism for colistin-dependent growth may be a mutation of lipid A, which results in a defective cell membrane and osmotic trauma in the absence of colistin [33]. The clinical importance of these subpopulations is yet to be determined.

In conclusion, our results indicate a risk of selective growth of colistin-resistant subpopulations with colistin monotherapy against $A$. baumannii and that combining colistin with rifampicin could be a way to prevent this occurrence.

\section{Compliance with Ethical Standards}

Funding This study was supported by grants from Region Östergötland, Sweden (Grant number LIO 281311 and LIO 449901). The funders had no role in study design, data collection and interpretation, or the decision to submit the work for publication.

Conflict of Interest The authors declare that they have no conflict of interest.

Ethical approval This article does not contain any studies with human participants or animals performed by any of the authors. The study was performed on bacterial isolates and did not involve any human or animal material. All isolates had been obtained as part of routine clinical care and patient identity had been decoded thereafter.

Informed consent For this type of study formal consent is not required.

Open Access This article is distributed under the terms of the Creative Commons Attribution 4.0 International License (http:// creativecommons.org/licenses/by/4.0/), which permits unrestricted use, distribution, and reproduction in any medium, provided you give appropriate credit to the original author(s) and the source, provide a link to the Creative Commons license, and indicate if changes were made.

Open Access This article is distributed under the terms of the Creative Commons Attribution 4.0 International License (http:// creativecommons.org/licenses/by/4.0/), which permits unrestricted use, distribution, and reproduction in any medium, provided you give appropriate credit to the original author(s) and the source, provide a link to the Creative Commons license, and indicate if changes were made.

\section{References}

1. Peleg AY, Seifert H, Paterson DL (2008) Acinetobacter baumannii: emergence of a successful pathogen. Clin Microbiol Rev 21:538 582

2. European Centre for Disease Prevention and Control (2014) Antimicrobial resistance surveillance in Europe 2013. Annual Report of the European Antimicrobial Resistance Surveillance Network (EARS-Net). ECDC, Stockholm

3. Falagas ME, Kasiakou SK (2005) Colistin: the revival of polymyxins for the management of multidrug-resistant gram-negative bacterial infections. Clin Infect Dis 40:1333-1341

4. Cai Y, Chai D, Wang R, Liang B, Bai N (2012) Colistin resistance of Acinetobacter baumannii: clinical reports, mechanisms and antimicrobial strategies. J Antimicrob Chemother 67:1607-1615

5. Ko KS, Suh JY, Kwon KT, Jung SI, Park KH, Kang CI, Chung DR, Peck KR, Song JH (2007) High rates of resistance to colistin and polymyxin B in subgroups of Acinetobacter baumannii isolates from Korea. J Antimicrob Chemother 60:1163-1167 
6. Falagas ME, Makris GC, Dimopoulos G, Matthaiou DK (2008) Heteroresistance: a concern of increasing clinical significance? Clin Microbiol Infect 14:101-104

7. Drlica K, Zhao X (2007) Mutant selection window hypothesis updated. Clin Infect Dis 44:681-688

8. Drlica K (2003) The mutant selection window and antimicrobial resistance. J Antimicrob Chemother 52:11-17

9. Cai Y, Li R, Liang B, Bai N, Liu Y, Wang R (2010) In vitro antimicrobial activity and mutant prevention concentration of colistin against Acinetobacter baumannii. Antimicrob Agents Chemother 54:3998-3999

10. Cai Y, Yang J, Kan Q, Nie X, Wang R, Liang B, Bai N (2012) Mutant prevention concentration of colistin alone and in combination with levofloxacin or tobramycin against multidrug-resistant Acinetobacter baumannii. Int J Antimicrob Agents 40:477-478

11. Li J, Nation RL, Owen RJ, Wong S, Spelman D, Franklin C (2007) Antibiograms of multidrug-resistant clinical Acinetobacter baumannii: promising therapeutic options for treatment of infection with colistin-resistant strains. Clin Infect Dis 45:594-598

12. Magiorakos AP, Srinivasan A, Carey RB, Carmeli Y, Falagas ME, Giske CG, Harbarth S, Hindler JF, Kahlmeter G, Olsson-Liljequist B, Paterson DL, Rice LB, Stelling J, Struelens MJ, Vatopoulos A, Weber JT, Monnet DL (2012) Multidrug-resistant, extensively drug-resistant and pandrug-resistant bacteria: an international expert proposal for interim standard definitions for acquired resistance. Clin Microbiol Infect 18:268-281

13. European Committee on Antimicrobial Susceptibility Testing (EUCAST). [On-line, EUCAST homepage], www.eucast.org. Accessed 5 May 2016

14. van Ingen J, Aarnoutse RE, Donald PR, Diacon AH, Dawson R, Plemper van Balen G, Gillespie SH, Boeree MJ (2011) Why do we use $600 \mathrm{mg}$ of rifampicin in tuberculosis treatment? Clin Infect Dis 52:194-199

15. The Swedish Reference Group for Antibiotics (SRGA). [On-line, SRGA homepage], www.sls.se/raf. Accessed 5 May 2016

16. Vidaillac C, Benichou L, Duval RE (2012) In vitro synergy of colistin combinations against colistin-resistant Acinetobacter baumannii, Pseudomonas aeruginosa, and Klebsiella pneumoniae isolates. Antimicrob Agents Chemother 56:4856-4861

17. Gordon NC, Png K, Wareham DW (2010) Potent synergy and sustained bactericidal activity of a vancomycin-colistin combination versus multidrug-resistant strains of Acinetobacter baumannii. Antimicrob Agents Chemother 54:5316-5322

18. Li J, Rayner CR, Nation RL, Owen RJ, Spelman D, Tan KE, Liolios L (2006) Heteroresistance to colistin in multidrug-resistant Acinetobacter baumannii. Antimicrob Agents Chemother 50: 2946-2950

19. Rodriguez CH, Bombicino K, Granados G, Nastro M, Vay C, Famiglietti A (2009) Selection of colistin-resistant Acinetobacter baumannii isolates in postneurosurgical meningitis in an intensive care unit with high presence of heteroresistance to colistin. Diagn Microbiol Infect Dis 65:188-191

20. Durante-Mangoni E, Signoriello G, Andini R, Mattei A, De Cristoforo M, Murino P, Bassetti M, Malacarne P, Petrosillo N, Galdieri N, Mocavero P, Corcione A, Viscoli C, Zarrilli R, Gallo C, Utili R (2013) Colistin and rifampicin compared with colistin alone for the treatment of serious infections due to extensively drug-resistant Acinetobacter baumannii: a multicenter, randomized clinical trial. Clin Infect Dis 57:349-358

21. Falagas ME, Rafailidis PI, Kasiakou SK, Hatzopoulou P, Michalopoulos A (2006) Effectiveness and nephrotoxicity of colistin monotherapy vs. colistin-meropenem combination therapy for multidrug-resistant Gram-negative bacterial infections. Clin Microbiol Infect 12:1227-1230

22. McLaughlin M, Advincula MR, Malczynski M, Qi C, Bolon M, Scheetz MH (2013) Correlations of antibiotic use and carbapenem resistance in enterobacteriaceae antimicrob. Agents Chemother 57: $5131-5133$

23. Giamarellos-Bourboulis EJ, Xirouchaki E, Giamarellou H (2001) Interactions of colistin and rifampin on multidrug-resistant Acinetobacter baumannii. Diagn Microbiol Infect Dis 40:117-120

24. Biswas S, Brunel JM, Dubus JC, Reynaud-Gaubert M, Rolain JM (2012) Colistin: an update on the antibiotic of the 21st century. Expert Rev Anti Infect Ther 10:917-934

25. Giannouli M, Di Popolo A, Durante-Mangoni E, Bernardo M, Cuccurullo S, Amato G, Tripodi MF, Triassi M, Utili R, Zarrilli R (2012) Molecular epidemiology and mechanisms of rifampicin resistance in Acinetobacter baumannii isolates from Italy. Int $\mathrm{J}$ Antimicrob Agents 39:58-63

26. Qureshi ZA, Hittle LE, O'Hara JA, Rivera JI, Syed A, Shields RK, Pasculle AW, Ernst RK, Doi Y (2015) Colistin-resistant Acinetobacter baumannii: beyond carbapenem resistance. Clin Infect Dis 60:1295-1303

27. López-Rojas R, McConnell MJ, Jiménez-Mejías ME, DomínguezHerrera J, Fernández-Cuenca F, Pachóna J (2013) Colistin resistance in a clinical Acinetobacter baumannii strain appearing after colistin treatment: effect on virulence and bacterial fitness. Antimicrob Agents Chemother 57:4587-4589

28. Bulitta JB, Yang JC, Yohonn L, Ly NS, Brown SV, D'Hondt RE, Jusko WJ, Forrest A, Tsuji BT (2010) Attenuation of colistin bactericidal activity by high inoculum of Pseudomonas aeruginosa characterized by a new mechanism-based population pharmacodynamic model. Antimicrob Agents Chemother 54:2051-2062

29. Lee SY, Shin JH, Lee K, Joo MY, Park KH, Shin MG, Suh SP, Ryang DW, Kim SH (2013) Comparison of the Vitek2, MicroScan, and Etest methods with the agar dilution method in assessing colistin susceptibility of bloodstream isolates of Acinetobacter species from a Korean university hospital. J Clin Microbiol 51:1924-1926

30. Maalej SM, Meziou MR, Rhimi FM, Hammami A (2011) Comparison of disc diffusion, Etest and agar dilution for susceptibility testing of colistin against Enterobacteriaceae. Lett Appl Microbiol 53:546-551

31. Arroyo LA, García-Curiel A, Pachón-Ibañez ME, Llanos AC, Ruiz M, Pachón J, Aznar J (2005) Reliability of the E-test method for detection of colistin resistance in clinical isolates of Acinetobacter baumannii. J Clin Microbiol 43:903-905

32. Hindler JA, Humphries RM (2013) Colistin MIC variability by method for contemporary clinical isolates of multidrug-resistant Gram-negative bacilli. J Clin Microbiol 51:1678-1684

33. Hawley J, Murray C (2007) Development of colistin-dependent Acinetobacter baumannii-Acinetobacter calcoaceticus complex. Antimicrob Agents Chemother 51:4529-4530 